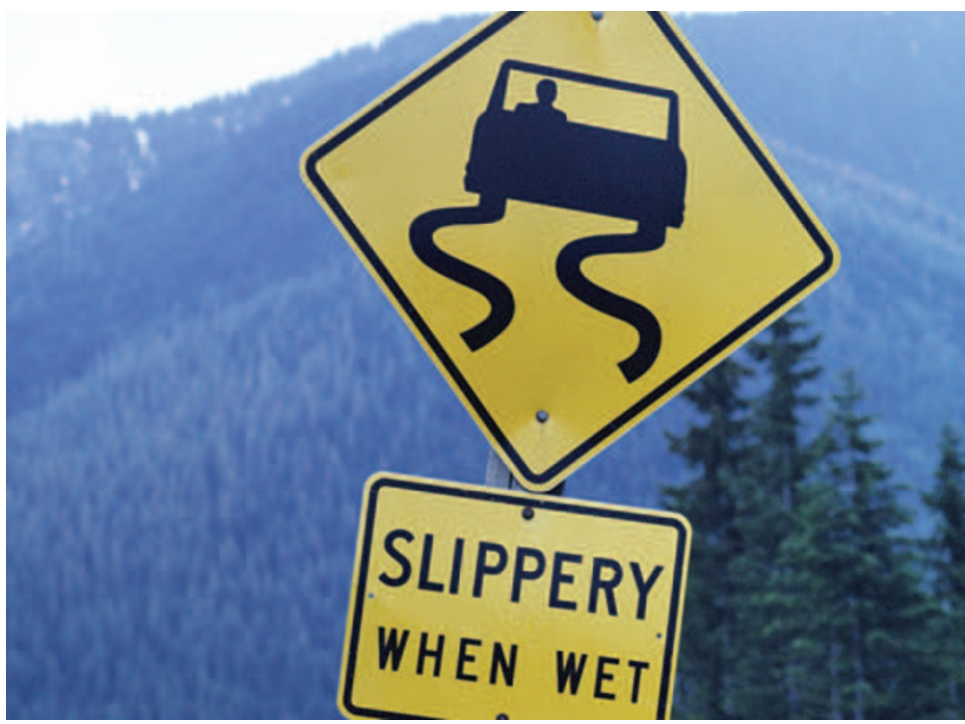

IMMUNOLOGY

\title{
Early warning
}

The production of interferon- $\gamma$ (IFN $\gamma$ ) is required for an effective, early (innate) immune response to tumour growth. A recent paper in Nature Medicine has identified that a novel subset of immune cells is able to produce this cytokine and kill tumour cells.

Dendritic cells (DCs) are an essential part of the immune system, as they help to activate lymphocytes in response to infection or tumour progression (an adaptive response), but part of the innate immune response to tumour development is mediated by natural killer (NK) cells. Recent studies indicate that there is substantial crosstalk between DCs and NK cells, so Taieb et al. studied the anti-tumour response in mice with B16F10 melanoma lung metastases. The authors had previously noted that imatinib mesylate (Glivec) generates an anti-tumour response to these metastases that is mediated by NK cells. In this study, combining imatinib mesylate with interleukin-2 (IL-2) was found to increase this anti-tumour response. It also increased the number of DCs infiltrating the tumours, and most of these cells expressed B220, NK1.1 and other NK-cell markers. Consistent with these cells being a new subset of DCs, about $50 \%$ also expressed major histocompatibilty complex (MHC) class II and could be detected in mice lacking lymphocytes and NK cells (mice lacking recombination-activating gene 2 (RAG2) and the common-cytokinereceptor $\gamma$-chain $\left(\gamma_{c}\right)$ ).

When isolated from mice treated with imatinib mesylate and IL-2, these DCs mediated TRAIL (tumour-necrosis factor (TNF)related apoptosis-inducing ligand)dependent tumour-cell lysis. Expression of TRAIL is induced by IFN $\gamma$, and when stimulated with either tumour cells or imatinib mesylate and IL-2, these DCs produced large amounts of IFN $\gamma$, hence their name - IFN-producing killer DCs (IKDCs). Importantly, IKDCs controlled tumour-cell growth when transferred to mice lacking RAG2 and $\gamma_{c}$, indicating that IKDCs induce an effective anti-tumour response in vivo.

The authors conclude that IKDCs might be an important link between the innate and adaptive immune response to tumour growth.

Karen Honey,

Senior Editor, Nature Reviews Immunology

ORIGINAL RESEARCH PAPER Taieb, J. et al. A novel dendritic cell subset involved in tumor immunosurveillance. Nature Med. 29 Jan 2006 (doi:10.1038/nm1356)

\section{Trial Watch}

\section{ASSESSING RADIATION EXPOSURE}

Clinical biomarkers of radiation exposure would be useful both in assessing exposure to radiation due to occupation, the environment or industrial accidents, and as early markers of clinical response to radiation therapy for cancer. Using a recently developed, robust, high-throughput technique, Cynthia Ménard and colleagues have shown that the composition of the serum proteome of cancer patients changes with ionizing radiation treatment.

Serum was collected before and during a course of radiation therapy from 68 patients who were diagnosed with cancer. The study population covered a wide range of diagnoses, body sites, and doses of radiotherapy. Aliquots of the fresh-frozen unfractionated sera were subjected to highresolution surface-enhanced laser desorption and ionization time of flight (SELDI-TOF) mass-spectrometry analysis to generate proteomic profiles using an immobilized metal ionaffinity chromatography nickel-affinity chip surface. These profiles were then analysed for unique biomarker signatures using supervised classification techniques.

The first question that the authors wanted to address was can proteomic profiles distinguish between unexposed and radiation-exposed patient serum? A total of 67 samples of unexposed sera were compared with 68 samples of exposed sera. The various classification methods that were used all showed close to $100 \%$ specificity and sensitivity in distinguishing the unexposed from the radiation-exposed samples.

Next, the authors tested whether the proteomic profiles could distinguish the high dose-volume from the low dosevolume radiation-exposure samples. The predictive results showed good performance in distinguishing between these two groups, with a sensitivity between $83 \%$ and $100 \%$, and a specificity between $91 \%$ and $100 \%$. However, the authors point out that these data must be interpreted with caution, given the small sample size and the lack of a patient-control study design.

Mass-spectrometry-based protein identification was then done on the pooled radiation-exposed and pooled unexposed sera to see if any specific protein fragments could be identified that are altered on radiation exposure. Out of 192 albuminbound protein fragments that were analysed in the two pooled sera sets, 82 were uniquely identified in the samples that had not been exposed to radiation, and 23 were uniquely identified in the radiation-exposed sera. One of the entities identified by this approach was a fragment of interleukin-6 (IL-6) that was found uniquely in the post-exposure sera. This finding is consistent with known changes in transcription after ionizing radiation, in which the nuclear factor- $\kappa \mathrm{B}$ pathway is activated - IL- 6 is one of the genes induced by this pathway.

The authors envisage that such a high-throughput blood test could be used to create personalized measures of clinical response to radiation therapy.

ORIGINAL RESEARCH PAPER Ménard, C. et al. Discovering clinical biomarkers of ionizing radiation exposure with serum proteomic analysis. Cancer Res. 66, 1844-1850 (2006) 